\title{
Risk Factors for Mortality Among Mechanically Ventilated Patients Requiring Pleural Drainage
}

\author{
Sojung Park (1)', Won-Young Kim², Moon Seong Baek $\mathbb{D}^{2}$ \\ 'Division of Pulmonary and Critical Care Medicine, Department of Internal Medicine, Mokdong Hospital, College of Medicine, Ewha Womans \\ University, Seoul, Republic of Korea; ${ }^{2}$ Department of Internal Medicine, Chung-Ang University Hospital, Chung-Ang University College of Medicine, \\ Seoul, Republic of Korea \\ Correspondence: Moon Seong Baek, Email wido2I@cau.ac.kr
}

\begin{abstract}
Purpose: Pleural effusions are common in mechanically ventilated patients. However, the risk factors for poor outcomes after pleural drainage are poorly understood. This study aimed to identify factors that were associated with in-hospital mortality among mechanically ventilated patients who underwent pleural drainage.

Methods: This retrospective study evaluated 82 consecutive patients who required chest tubes during mechanical ventilation at two university-affiliated hospitals in Korea between January 2015 and June 2020.

Results: The median age was 76 years (interquartile range [IQR]: 64-84 years), and the median SOFA score was 11 (IQR: 7-13). Intensive care unit admission was most commonly because of pneumonia $(\mathrm{n}=44,53.7 \%)$ and 60 patients $(77.9 \%)$ had exudative pleural effusions. During pleural drainage, the $\mathrm{PaO}_{2} / \mathrm{FiO}_{2}$ was 210 (IQR: 153-253); 45 patients (54.9\%) were receiving vasopressors, and 31 patients $(37.8 \%)$ were receiving continuous renal replacement therapy (CRRT). The multivariable regression analysis revealed that poor overall survival was independently associated with receiving vasopressors (adjusted hazard ratio [aHR]: 3.81, 95\% confidence interval $[\mathrm{CI}]: 1.65-8.81, \mathrm{p}=0.002)$ and receiving CRRT (aHR: 5.48, 95\% CI: 2.29-13.12, $\mathrm{p}<0.001$ ). $\mathrm{The} \mathrm{PaO}_{2} / \mathrm{FiO}_{2}$ ratio was relatively stable through the third day of pleural drainage among survivors but decreased among non-survivors. The vasopressor dose decreased among survivors but remained relatively stable among non-survivors.

Conclusion: Among mechanically ventilated patients who required pleural drainage, use of vasopressors and CRRT was significantly associated with in-hospital mortality. On the third day of pleural drainage, the changes in $\mathrm{PaO}_{2} / \mathrm{FiO}_{2}$ and vasopressor dose were associated with in-hospital mortality.
\end{abstract}

Keywords: pleural fluid, drainage, mechanical ventilation, mortality

\section{Introduction}

Pleural effusions are common in patients who are treated in the intensive care unit (ICU). For example, a single-center study revealed that $27 \%$ of patients who were admitted to the ICU had pleural effusions that were identified via chest radiography. ${ }^{1}$ Another large observational study revealed that $7.7 \%$ of critically ill patients had pleural effusions. ${ }^{2}$ Furthermore, among critically ill patients, patients with pleural effusions had a higher rate of in-hospital mortality than patients without effusions (38.7\% vs $31.3 \%)^{2}$. Pleural fluid accumulation occurs when there is an imbalance in fluid production and drainage, which can be aggravated by mechanical ventilation (MV). ${ }^{3}$ The increase in pleural fluid leads to restrictive ventilatory defects that can cause hypoxemia, ${ }^{3-6}$ and is associated with various hemodynamic parameters. ${ }^{7}$

Some studies have evaluated the effects of pleural effusion drainage on oxygenation, respiratory mechanics, diaphragm function, and hemodynamics during MV. Razazi et al suggested that a large amount of pleural drainage $(>500 \mathrm{~mL})$ was associated with increases in respiratory system compliance and oxygenation, as the $\mathrm{PaO}_{2} / \mathrm{FiO}_{2}$ ratio increased from 191 to 250 at $24 \mathrm{~h}$ after effusion drainage. ${ }^{8}$ Another observational study revealed that moderate-to-large pleural effusion was associated with failure to achieve MV weaning. ${ }^{9}$ Umbrello et al also reported that draining pleural effusion during MV weaning helps improve diaphragmatic function. ${ }^{10}$ However, it is unclear whether these positive effects of pleural drainage are associated with survival among mechanically ventilated patients, and remains poorly 
understood the risk factor for mortality. Therefore, this study aimed to identify factors that were associated with inhospital mortality among mechanically ventilated patients who underwent pleural drainage.

\section{Methods}

\section{Study Design}

This retrospective study evaluated 82 consecutive adult patients ( $\geq 18$ years old) who required chest tubes during MV at two university-affiliated tertiary hospitals in the Republic of Korea between January 1, 2015 and December 31, 2020. Pleural effusions were identified based on daily chest radiography screening and were confirmed by a board-certified pulmonologist using ultrasonography. The decision to perform pleural effusion drainage was made by the pulmonologist and attending physician according to the following conditions: for patients with large pleural effusion ( $\geq 1 / 2$ hemithorax), or for patients with small to moderate effusion $(>10 \mathrm{~mm}$ and $<1 / 2$ hemithorax) for which diuretics or renal replacement therapy (RRT) are ineffective. ${ }^{11}$ To yield minimal safety margin, pleural drainage was attempted for patients with maximal inter-pleural separation of $>20 \mathrm{~mm}$ indicated by ultrasonography. ${ }^{12}$ Patients were excluded if they had undergone pleural drainage before or after the MV. The retrospective study protocol was approved by the institutional review board of Chung-Ang University Hospital (2011-010-19341), which waived the requirement for informed consent.

\section{Data Collection}

The patients' electronic medical records were searched to collect the following data from the time of the pleural drainage: age, sex, body mass index, Sequential Organ Failure Assessment (SOFA) score, Charlson comorbidity index, comorbidities, reasons for admission to the medical intensive care unit (MICU), duration of hospital stay, duration of MICU stay, duration of $\mathrm{MV}$, tracheostomy, vital signs, ventilator parameters $\left(\mathrm{FiO}_{2}\right.$, positive end-expiratory pressure [PEEP], tidal volume, and peak inspiratory pressure), arterial blood gas parameters ( $\mathrm{pH}, \mathrm{PaCO}_{2}, \mathrm{PaO}_{2}, \mathrm{HCO}_{3}$, and $\mathrm{SaO}_{2}$ ), $\mathrm{PaO}_{2} / \mathrm{FiO}_{2}$ ratio, and laboratory results (concentrations of protein, albumin, lactate dehydrogenase [LDH], as well as leukocyte count). The $\mathrm{PaO} 2 / \mathrm{FiO} 2$ ratio and vasopressor dose were recorded on days 1-3 before the pleural fluid drainage, at the time of drainage (baseline), and on days $1-3$ after the drainage.

The characteristics of the pleural effusion and intervention were: time from MV to drainage catheter insertion, duration of pleural drainage, need for vasopressors or continuous renal replacement therapy (CRRT), laterality (right, left, or bilateral), 24-h drainage volume, type of pleural drainage (percutaneous pigtail drainage or surgical thoracostomy), operator (interventional radiologist, cardiac surgeon, intensivist, or emergency medicine doctor), location (angiography room, MICU, or emergency room), complications (pneumothorax and subcutaneous emphysema), and pleural effusion findings (appearance, laboratory findings [pH, leukocyte and erythrocyte counts, proportion of neutrophils, and concentrations of protein, albumin, glucose, and $\mathrm{LDH}]$, and bacterial smear/culture findings).

Pleural effusions were classified as exudates or transudates using Light's criteria. ${ }^{13}$ Exudative pleural effusions fulfilled at least one of the following criteria and transudative effusions fulfilled none of the criteria: ratio of pleural-fluid protein to serum protein $>0.5$, ratio of pleural fluid LDH to serum LDH concentration $>0.6$, and pleural-fluid LDH concentration of $>2 / 3$ the upper limit of normal for serum. Parapneumonic effusion was defined as any pleural effusion secondary to pneumonia or lung abscess, which is characterized as an exudate with a predominance of neutrophils. ${ }^{14}$ Parapneumonic effusion was considered complicated effusion if the pleural fluid $\mathrm{pH}$ was $<7.2$, the glucose concentration was $<60 \mathrm{mg} / \mathrm{dL}$, or the $\mathrm{LDH}$ concentration was $>3 \times$ the upper limit of normal for serum. ${ }^{14,15}$ Empyema was defined based on the presence of bacteria or pus in the pleural space. The vasopressor doses were calculated using the following equation: norepinephrine equivalents $=$ norepinephrine + epinephrine $+($ phenylephrine $/ 10)+($ dopamine $/ 100)+($ metar$\operatorname{aminol} / 8)+($ vasopressin $\times 2.5)+($ angiotensin $\mathrm{II} \times 10) .{ }^{16}$

\section{Statistical Analyses}

Categorical variables are presented as numbers (\%) and were compared using the Pearson's chi-squared test or Fisher's exact test. Continuous variables are presented as median (interquartile range [IQR]) or mean \pm standard deviation and were compared using the Student's $t$ test or the Mann-Whitney $U$-test. Univariate and multivariable Cox proportional 
hazard regression analyses were performed to identify factors that were significantly associated with cumulative survival. Significant variables in the univariate analyses $(p<0.05)$ and clinically significant variables were entered into the multivariable analysis, with the results reported as hazard ratios (HRs) and $95 \%$ confidence intervals (CIs). KaplanMeier curves for cumulative survival were created and compared using the Log rank test. The effects of pleural drainage on oxygenation and hemodynamics were evaluated using one-way analysis of variance for repeated measures to compare the $\mathrm{PaO}_{2} / \mathrm{FiO}_{2}$ ratio and vasopressor dose among survivors and non-survivors. Inter-group comparisons at each time point were performed using the paired-samples Student $t$ test. Differences were considered statistically significant at p-values of $<0.05$ and all analyses were performed using SPSS software (version 26.0; IBM Corporation, Armonk, NY, USA).

\section{Results}

The study included 82 consecutive patients who underwent pleural drainage catheter insertion during MV. The baseline patient characteristics are shown in Table 1. The median age was 76 years (IQR: 64-84 years) and 52 patients (63.42\%) were male. The median SOFA score was 11 (IQR: 7-13) and 90.2\% of the patients had comorbidities. The common reasons for admission to the ICU were pneumonia $(n=44,53.7 \%)$, cardiovascular disorders $(n=14,17.1 \%)$, and postcardiac arrest care $(\mathrm{n}=10,12.2 \%)$. The median duration of ICU stay was 42 days (IQR: 16-44 days) and tracheostomy was performed in 39 patients (47.6\%). The median $\mathrm{FiO}_{2}$ was 0.40 (IQR: 0.35-0.50) with a median PEEP of $8 \mathrm{cmH}_{2}$ O (IQR: 5-8 cmH $\mathrm{c}_{2} \mathrm{O}$ ) (Table 2). The median $\mathrm{PaO}_{2}$ was $85 \mathrm{mmHg}$ (IQR: $73-98 \mathrm{mmHg}$ ) and the median $\mathrm{PaO}_{2} / \mathrm{FiO}_{2}$ was 210 (IQR: 153-253).

Table I Baseline Patient Characteristics

\begin{tabular}{|c|c|c|c|c|}
\hline Variables & Total $(n=82)$ & Survivors $(n=40)$ & Non-Survivors $(n=42)$ & P-value \\
\hline Age (years) & $76(64-84)$ & $77(64-85)$ & $73(64-82)$ & 0.263 \\
\hline Male sex & $52(63.4)$ & $25(62.5)$ & $27(64.3)$ & 1.000 \\
\hline Body mass index $\left(\mathrm{kg} / \mathrm{m}^{2}\right)$ & $20.7(18.7-24.8)$ & $22.0(19.5-25.2)$ & $20.0(18.3-23.5)$ & 0.092 \\
\hline SOFA score & II (7-13) & $10(6-14)$ & II (8-13) & 0.421 \\
\hline Charlson comorbidity index & $5(4-7)$ & $6(4-7)$ & $5(3-8)$ & 0.737 \\
\hline Comorbidities & $74(90.2)$ & $35(87.5)$ & $39(92.9)$ & 0.477 \\
\hline Diabetes mellitus & $31(37.8)$ & $13(32.5)$ & $18(42.9)$ & 0.334 \\
\hline Hypertension & $44(53.7)$ & $22(55.0)$ & $22(52.4)$ & 0.812 \\
\hline Chronic lung disease & $10(12.2)$ & $5(12.5)$ & $5(11.9)$ & 1.000 \\
\hline Chronic kidney disease & II (I3.4) & $5(12.5)$ & $6(14.3)$ & 0.813 \\
\hline Chronic liver disease & $5(6.1)$ & $3(7.5)$ & $2(4.8)$ & 0.672 \\
\hline Cardiovascular disorders & $22(26.8)$ & $13(32.5)$ & $9(21.4)$ & 0.258 \\
\hline Neurological disorders & $23(28.0)$ & $14(35.0)$ & $9(21.4)$ & 0.262 \\
\hline Malignancy & $21(25.6)$ & $9(22.5)$ & $12(28.6)$ & 0.529 \\
\hline Reasons for ICU admission & & & & $0.4 I I$ \\
\hline Pneumonia & $44(53.7)$ & $22(55.0)$ & $22(52.4)$ & \\
\hline Sepsis & $5(6.1)$ & $\mathrm{I}(2.5)$ & $4(9.5)$ & \\
\hline Respiratory disorders & $7(8.5)$ & $5(12.5)$ & $2(4.8)$ & \\
\hline Cardiovascular disorders & $14(17.1)$ & $7(17.5)$ & $7(16.7)$ & \\
\hline Post-cardiac arrest care & $10(12.2)$ & $5(12.5)$ & $5(11.9)$ & \\
\hline Neurological disorders & $2(2.4)$ & $0(0.0)$ & $2(4.8)$ & \\
\hline Length of stay, days & $42(25-74)$ & $53(35-87)$ & $33(22-53)$ & 0.006 \\
\hline ICU stay, days & $25(16-44)$ & $25(14-46)$ & $27(18-43)$ & 0.553 \\
\hline Duration of MV, days & $21(11-40)$ & $19(8-41)$ & $24(15-39)$ & 0.172 \\
\hline Tracheostomy & $39(47.6)$ & $21(52.5)$ & $18(42.9)$ & 0.507 \\
\hline
\end{tabular}

Note: Values are presented as median (interquartile range) or number (\%).

Abbreviations: SOFA, Sequential Organ Failure Assessment; ICU, intensive care unit; MV, mechanical ventilation. 
Table 2 Ventilator Parameters and Laboratory Findings at the Pleural Effusion Drainage

\begin{tabular}{|c|c|c|c|c|}
\hline Variables & Total $(n=82)$ & Survivors $(n=40)$ & Non-Survivors $(n=42)$ & P-value \\
\hline \multicolumn{5}{|l|}{ Vital signs } \\
\hline MAP $(\mathrm{mmHg})$ & $79(72-89)$ & 79 (72-91) & 79 (72-87) & 0.784 \\
\hline Heart rate $(/ \mathrm{min})$ & $91(76-104)$ & $86(75-102)$ & $96(79-108)$ & 0.161 \\
\hline Respiratory rate (/min) & $23(20-25)$ & $23(20-24)$ & $22(20-27)$ & 0.724 \\
\hline Body temperature $\left({ }^{\circ} \mathrm{C}\right)$ & $36.6(36.2-37.1)$ & $36.9(36.5-37.2)$ & $36.4(36.1-36.8)$ & 0.001 \\
\hline Oxygen saturation (\%) & $98(96-100)$ & $99(96-100)$ & $98(96-100)$ & 0.277 \\
\hline \multicolumn{5}{|l|}{ Ventilation parameters } \\
\hline $\mathrm{FiO}_{2}$ & $0.40(0.35-0.50)$ & $0.43(0.35-0.50)$ & $0.40(0.40-0.53)$ & 0.579 \\
\hline PEEP $\left(\mathrm{cmH}_{2} \mathrm{O}\right)$ & $8(5-8)$ & $7(5-9)$ & $8(6-8)$ & 0.806 \\
\hline Tidal volume $(\mathrm{mL})$ & $400(330-458)$ & $394(326-459)$ & $400(330-458)$ & 0.788 \\
\hline Peak inspiratory pressure $\left(\mathrm{cmH}_{2} \mathrm{O}\right)$ & $19(16-23)$ & $19(15-23)$ & $20(16-24)$ & 0.507 \\
\hline \multicolumn{5}{|l|}{ Arterial blood gases } \\
\hline $\mathrm{pH}$ & $7.4 \mid(7.36-7.45)$ & $7.44(7.39-7.45)$ & $7.39(7.35-7.44)$ & 0.140 \\
\hline $\mathrm{PaCO}_{2}(\mathrm{mmHg})$ & $40(35-45)$ & $39(36-49)$ & $4 \mid(34-44)$ & 0.565 \\
\hline $\mathrm{PaO}_{2}(\mathrm{mmHg})$ & $85(73-98)$ & $85(74-97)$ & $84(73-105)$ & 0.770 \\
\hline $\mathrm{HCO}_{3}^{-}(\mathrm{mmol} / \mathrm{L})$ & $24(2 I-28)$ & $25(23-3 I)$ & $23(2 \mathrm{I}-27)$ & 0.063 \\
\hline $\mathrm{SaO}_{2}(\%)$ & $96(95-98)$ & $96(95-98)$ & $96(94-98)$ & 0.824 \\
\hline $\mathrm{PaO}_{2} / \mathrm{FiO}_{2}$ ratio & $210(153-253)$ & $210(156-253)$ & $211(144-253)$ & 0.919 \\
\hline \multicolumn{5}{|l|}{ Laboratory results } \\
\hline White blood cells $\left(\times 10^{9} / \mathrm{L}\right)$ & II.4 (7.3-15.7) & $10.0(6.7-14.2)$ & $12.5(8.5-16.6)$ & 0.109 \\
\hline Hemoglobin $(g / d L)$ & $9.0(8.2-10.1)$ & $9.0(7.9-9.9)$ & $9.0(8.3-10.2)$ & 0.183 \\
\hline Platelets $\left(\times 10^{9} / \mathrm{L}\right)$ & $149(64-210)$ & $169(107-247)$ & $88(57-161)$ & 0.001 \\
\hline Total protein $(\mathrm{g} / \mathrm{dL})$ & $5.3(4.6-5.7)$ & $5.4(4.7-5.9)$ & $5.0(4.6-5.6)$ & 0.061 \\
\hline Albumin $(\mathrm{g} / \mathrm{dL})$ & $2.4(2.1-2.7)$ & $2.4(2.1-2.7)$ & $2.4(2.0-2.7)$ & 0.331 \\
\hline Total bilirubin $(\mathrm{mg} / \mathrm{dL})$ & $0.7(0.5-1.4)$ & $0.6(0.3-0.9)$ & $1.0(0.6-2.5)$ & 0.003 \\
\hline Aspartate aminotransferase (IU/L) & $37(23-96)$ & $29(22-64)$ & $47(3|-| 7 \mid)$ & 0.017 \\
\hline Alanine aminotransferase (IU/L) & $28(13-53)$ & $26(11-45)$ & $30(14-90)$ & 0.371 \\
\hline Blood urea nitrogen $(\mathrm{mg} / \mathrm{dL})$ & $27(18-38)$ & $25(18-38)$ & $28(|7-4|)$ & 0.766 \\
\hline Serum creatinine $(\mathrm{mg} / \mathrm{dL})$ & $1.01(0.63-1.59)$ & $1.05(0.64-1.59)$ & $0.98(0.63-1.60)$ & 0.886 \\
\hline Serum sodium (mmol/L) & $138(|34-| 4 \mid)$ & $138(134-142)$ & $138(134-140)$ & 0.488 \\
\hline Serum potassium $(\mathrm{mmol} / \mathrm{L})$ & $3.9(3.6-4.2)$ & $3.8(3.7-4.2)$ & $4.0(3.5-4.3)$ & 0.933 \\
\hline C-reactive protein $(\mathrm{mg} / \mathrm{dL})$ & $10 \mid(47-172)$ & $72(45-120)$ & $130(67-198)$ & 0.018 \\
\hline Lactic dehydrogenase (IU/L) & $306(233-379)$ & $300(233-345)$ & $308(23 \mathrm{I}-580)$ & 0.253 \\
\hline
\end{tabular}

Note: Values are presented as median (interquartile range) or number (\%).

Abbreviations: MAP, mean arterial pressure; $\mathrm{FiO}_{2}$, fraction of inspired oxygen; $\mathrm{PEEP}$, positive end-expiratory pressure; $\mathrm{PaCO}$, partial pressure of carbon dioxide; $\mathrm{PaO}$, partial pressure of oxygen; $\mathrm{HCO}_{3}{ }^{-}$, bicarbonate; $\mathrm{SaO}_{2}$, oxygen saturation.

The pleural drainage-related variables and pleural effusion findings are shown in Table 3. At the time of pleural drainage, 45 patients $(54.9 \%)$ were receiving vasopressors and 31 patients $(37.8 \%)$ were receiving CRRT. The median 24-h drainage volume was $1000 \mathrm{~mL}$ (IQR: $644-1463 \mathrm{~mL}$ ). Procedure-related complications occurred in 3 patients (3.7\%), which involved pneumothorax in 2 patients and subcutaneous emphysema in 1 patient. The effusions were classified as exudative in 60 patients $(77.9 \%)$ and complicated pleural effusions in 17 patients $(22.1 \%)$. Relative to survivors, non-survivors were significantly more likely to receive vasopressors $(66.7 \%$ vs $42.5 \%, \mathrm{p}=0.028)$ and CRRT $(59.5 \%$ vs $15.0 \%, \mathrm{p}<0.001)$.

Table 4 shows the multivariable regression analysis of factors that were associated with death. Significantly shorter overall survival was independently associated with use of vasopressors (aHR: 3.81, 95\% CI: $1.65-8.81, \mathrm{p}=0.002$ ) and CRRT (aHR: 5.48, 95\% CI: 2.29-13.12, p<0.001). Figure 1 shows the Kaplan-Meier curves, which revealed that nonuse of CRRT was associated with significantly longer median overall survival, relative to CRRT use (non-CRRT: 147 days [95\% CI: $62-232$ days] vs CRRT: 36 days [95\% CI: $27-45$ days], $p<0.001)$. Furthermore, transudative effusion 
Table 3 Factors Related to Pleural Drainage and Characteristics of Pleural Effusion

\begin{tabular}{|c|c|c|c|c|}
\hline Variables & Total $(n=82)$ & Survivors $(n=40)$ & Non-Survivors $(n=42)$ & P-value \\
\hline Time from MV to pleural drainage (days) & $8(3-17)$ & $8(3-12)$ & $8(3-26)$ & 0.322 \\
\hline Duration of pleural drainage (days) & $14(8-2 \mid)$ & $17(7-28)$ & $13(8-17)$ & 0.129 \\
\hline Need for vasopressors & $45(54.9)$ & $17(42.5)$ & $28(66.7)$ & 0.028 \\
\hline CRRT & $31(37.8)$ & $6(15.0)$ & $25(59.5)$ & $<0.001$ \\
\hline Laterality of pleural drainage & & & & 0.818 \\
\hline Right & $32(39.0)$ & $15(37.5)$ & $17(40.5)$ & \\
\hline Left & $22(26.8)$ & $12(30.0)$ & $10(23.8)$ & \\
\hline Bilateral & $28(34.1)$ & $13(32.5)$ & $15(35.7)$ & \\
\hline 24-h drainage volume $(\mathrm{mL})$ & $1000(644-1463)$ & $995(618-1353)$ & $1020(665-1533)$ & 0.420 \\
\hline Type of pleural drainage & & & & 0.474 \\
\hline Percutaneous pigtail drainage & $70(85.4)$ & $33(47.1)$ & $37(52.9)$ & \\
\hline Surgical thoracostomy & $12(14.6)$ & $7(58.3)$ & $5(4 I .7)$ & \\
\hline Operator & & & & 0.622 \\
\hline Interventional radiologist & $50(61.7)$ & $23(59.0)$ & $27(64.3)$ & \\
\hline Cardiac surgeon & $12(14.8)$ & $7(17.9)$ & $5(11.9)$ & \\
\hline Intensivist & $18(22.2)$ & $8(20.5)$ & $10(23.8)$ & \\
\hline Emergency medicine doctor & $\mathrm{I}(\mathrm{I} .2)$ & I (2.6) & $0(0.0)$ & \\
\hline Location & & & & 0.325 \\
\hline Angiography room & $50(61.7)$ & $23(59.0)$ & $27(64.3)$ & \\
\hline ICU & $29(35.8)$ & $14(35.9)$ & $15(35.7)$ & \\
\hline Emergency room & $2(2.5)$ & $2(5.1)$ & $0(0.0)$ & \\
\hline Complications & & & & 0.227 \\
\hline Pneumothorax & $2(2.4)$ & $0(0)$ & $2(4.8)$ & \\
\hline Subcutaneous emphysema & $\mathrm{I}(1.2)$ & I (2.5) & $0(0)$ & \\
\hline Exudate vs transudate $^{\dagger}$ & & & & 0.151 \\
\hline Exudate & $60(77.9)$ & 27 (7I.I) & $33(84.6)$ & \\
\hline Transudate & $17(22.1)$ & II (28.9) & $6(15.4)$ & \\
\hline Complicated pleural effusion $n^{\ddagger}$ & $17(22.1)$ & $6(15.8)$ & II (28.2) & 0.189 \\
\hline Empyema & $6(7.9)$ & I (2.7) & $5(12.8)$ & 0.201 \\
\hline \multicolumn{5}{|l|}{ Pleural effusion findings } \\
\hline $\mathrm{pH}$ & $7.38(7.18-7.62)$ & $7.42(7.22-7.68)$ & $7.36(7.13-7.56)$ & 0.234 \\
\hline $\operatorname{RBCs}\left(\mathrm{mm}^{3}\right)$ & $3000(1000-8425)$ & $2275(1000-7558)$ & $3300(1000-27,000)$ & 0.504 \\
\hline $\mathrm{WBC}\left(\mathrm{mm}^{3}\right)$ & $367(114-863)$ & $369(89-1434)$ & $367(15 \mid-620)$ & 0.951 \\
\hline Neutrophils (\%) & $42(15-79)$ & $31(15-73)$ & $53(14-90)$ & 0.204 \\
\hline Lymphocyte (\%) & $26(||-5 \mid)$ & $29(13-69)$ & $20(8-42)$ & 0.142 \\
\hline Protein $(g / d L)$ & $2.4(1.5-3.2)$ & $2.4(1.5-3.6)$ & $2.3(1.9-3.1)$ & 0.643 \\
\hline Albumin (g/dL) & $1.2(0.8-1.6)$ & $1.3(0.9-1.8)$ & $1.2(0.8-1.4)$ & 0.327 \\
\hline LDH (IU/L) & $192(127-345)$ & $160(113-270)$ & $253(|39-5| I)$ & 0.066 \\
\hline Glucose $(\mathrm{mg} / \mathrm{dL})$ & $140(110-175)$ & $138(120-175)$ & $142(100-176)$ & 0.585 \\
\hline Pleural fluid/serum protein ratio & $0.53(0.36-0.70)$ & $0.56(0.30-0.68)$ & $0.5 \mathrm{I}(0.4 \mathrm{I}-0.74)$ & 0.840 \\
\hline
\end{tabular}

Notes: Values are presented as median (interquartile range) or number (\%). 'Exudate fulfilled at least one of the following criteria and transudate did not fulfill any of the criteria: a $>0.5$ ratio of pleural-fluid protein to serum protein, a $>0.6$ ratio of pleural fluid LDH to serum LDH, and pleural-fluid LDH concentration $>2 / 3$ the upper limit of normal for serum. ${ }^{\ddagger}$ Parapneumonic effusion is considered complicated effusion if the pleural fluid $\mathrm{pH}$ is $<7.2$, the glucose concentration is $<60 \mathrm{mg} / \mathrm{dL}$, or the $\mathrm{LDH}$ concentration is $>3 \times$ the upper limit of normal for serum.

Abbreviations: MV, mechanical ventilation; CRRT, continuous renal replacement therapy; ICU, intensive care unit; RBC, red blood cell; WBC, white blood cell; LDH, lactate dehydrogenase.

was associated with significantly longer median overall survival, relative to exudative effusion (transudative: 85 days [95\% CI: 0-314 days] vs exudative: 24 days [95\% CI: 6-100 days], $\mathrm{p}=0.039$ ).

Figure 2 shows the changes in the $\mathrm{PaO}_{2} / \mathrm{FiO}_{2}$ ratio and vasopressor dose before and after pleural drainage (Supplementary Materials Tables $\mathrm{S} 1$ and $\underline{\mathrm{S} 2}$ ). After $24 \mathrm{~h}$ of pleural drainage, the mean $\mathrm{PaO}_{2} / \mathrm{FiO}_{2}$ ratio had risen from 206 to $245(\mathrm{p}<0.001$, Figure $2 \mathrm{~A})$ and the vasopressor dose had decreased from $0.10 \mu \mathrm{g} / \mathrm{kg} / \mathrm{min}$ to $0.07 \mu \mathrm{g} / \mathrm{kg} / \mathrm{min}$ 
Table 4 Univariate and Multivariable Regression Analyses of Factors Associated with Death

\begin{tabular}{|c|c|c|c|c|}
\hline Variables & $\begin{array}{l}\text { Univariate } \\
\text { HR }(95 \% \mathrm{Cl})\end{array}$ & P-value & $\begin{array}{l}\text { Multivariable } \\
\text { HR }(95 \% \mathrm{Cl})\end{array}$ & P-value \\
\hline Age (years) & $0.99(0.97-1.01)$ & 0.166 & & \\
\hline Sex & & 0.475 & & 0.050 \\
\hline Male & Reference & & Reference & \\
\hline Female & $0.79(0.40-1.52)$ & & $0.43(0.19-1.00)$ & \\
\hline Body mass index $\left(\mathrm{kg} / \mathrm{m}^{2}\right)$ & $0.96(0.89-1.04)$ & 0.313 & & \\
\hline SOFA score & $1.04(0.96-I .12)$ & 0.362 & & \\
\hline Charlson comorbidity index & $1.06(0.95-1.19)$ & 0.287 & & \\
\hline Tracheostomy & $0.35(0.18-0.68)$ & 0.002 & & \\
\hline Reasons for ICU admission & & 0.410 & & 0.024 \\
\hline Pneumonia & Reference & & Reference & \\
\hline Sepsis & $0.35(0.12-1.03)$ & & $0.32(0.10-1.08)$ & \\
\hline Respiratory disorders & $0.56(0.15-2.11)$ & & $1.77(0.36-8.66)$ & \\
\hline Cardiovascular disorders & $0.48(0.14-1.64)$ & & $0.15(0.03-0.66)$ & \\
\hline Post-cardiac arrest care & $0.20(0.04-1.14)$ & & $0.53(0.07-3.86)$ & \\
\hline Neurological disorders & $0.45(0.08-2.68)$ & & $0.79(0.11-5.59)$ & \\
\hline Exudate vs transudate & & 0.048 & & \\
\hline Transudate & Reference & & & \\
\hline Exudate & $2.62(1.01-6.80)$ & & & \\
\hline $\mathrm{PaO}_{2} / \mathrm{FiO}_{2}$ ratio & & 0.728 & & \\
\hline$>200$ & Reference & & & \\
\hline$\leq 200$ & $0.78(0.40-1.5 \mathrm{I})$ & & & \\
\hline$\leq 100$ & $1.04(0.36-3.02)$ & & & \\
\hline Vasopressors & & 0.003 & & 0.002 \\
\hline No & Reference & & Reference & \\
\hline Yes & $2.78(\mathrm{I} .4 \mathrm{I}-5.47)$ & & $3.8 \mathrm{I}(\mathrm{I} .65-8.8 \mathrm{I})$ & \\
\hline CRRT & & $<0.001$ & & $<0.001$ \\
\hline No & Reference & & Reference & \\
\hline Yes & $3.11(1.66-5.8 I)$ & & $5.48(2.29-13.12)$ & \\
\hline
\end{tabular}

Notes: The multivariable Cox regression analysis was adjusted for age, sex, body mass index, SOFA score, Charlson comorbidity index, tracheostomy, reasons for ICU admission, exudate vs transudate, $\mathrm{PaO}_{2} / \mathrm{FiO}_{2}$ ratio, vasopressors, and CRRT.

Abbreviations: HR, hazard ratio; $\mathrm{Cl}$, confidence interval; SOFA, Sequential Organ Failure Assessment; ICU, intensive care unit; CRRT, continuous renal replacement therapy.

( $\mathrm{p}=0.03$, Figure $2 \mathrm{~B}$ ). After day 3 of pleural drainage, the $\mathrm{PaO}_{2} / \mathrm{FiO}_{2}$ ratio tended to decrease among non-survivors, relative to among survivors (survivors: $263 \pm 100$ vs non-survivors: $229 \pm 104, \mathrm{p}=0.102$ ) (Figure $2 \mathrm{C}$ ). However, the vasopressor dose decreased among survivors but not among non-survivors (survivors: $0.01 \pm 0.04 \mu \mathrm{g} / \mathrm{kg} / \mathrm{min}$ vs nonsurvivors: $0.08 \pm 0.18 \mu \mathrm{g} / \mathrm{kg} / \mathrm{min}, \mathrm{p}=0.011$ ) (Figure 2D).

\section{Discussion}

This multicenter study revealed that among mechanically ventilated patients, pleural drainage was typically performed for patients with greater morbidity and was associated with a high mortality rate. In this patient population, mortality was related to the use of CRRT and vasopressors at the time of the drainage procedure, and exudative effusions were associated with poorer outcomes than transudative effusions. Furthermore, survival could be predicted by the $\mathrm{PaO}_{2}$ $/ \mathrm{FiO}_{2}$ ratio and vasopressor dose on the third day of pleural drainage. Therefore, changes in oxygenation parameters and the vasopressor dose should be carefully monitored among mechanically ventilated patients who require pleural drainage.

Pleural effusion may be a marker of disease severity and may predict mortality. ${ }^{17}$ For example, patients undergoing thoracentesis for malignant pleural effusion have a 30 -day mortality rate of $37 \% .{ }^{18}$ In addition, patients with cirrhosis and 

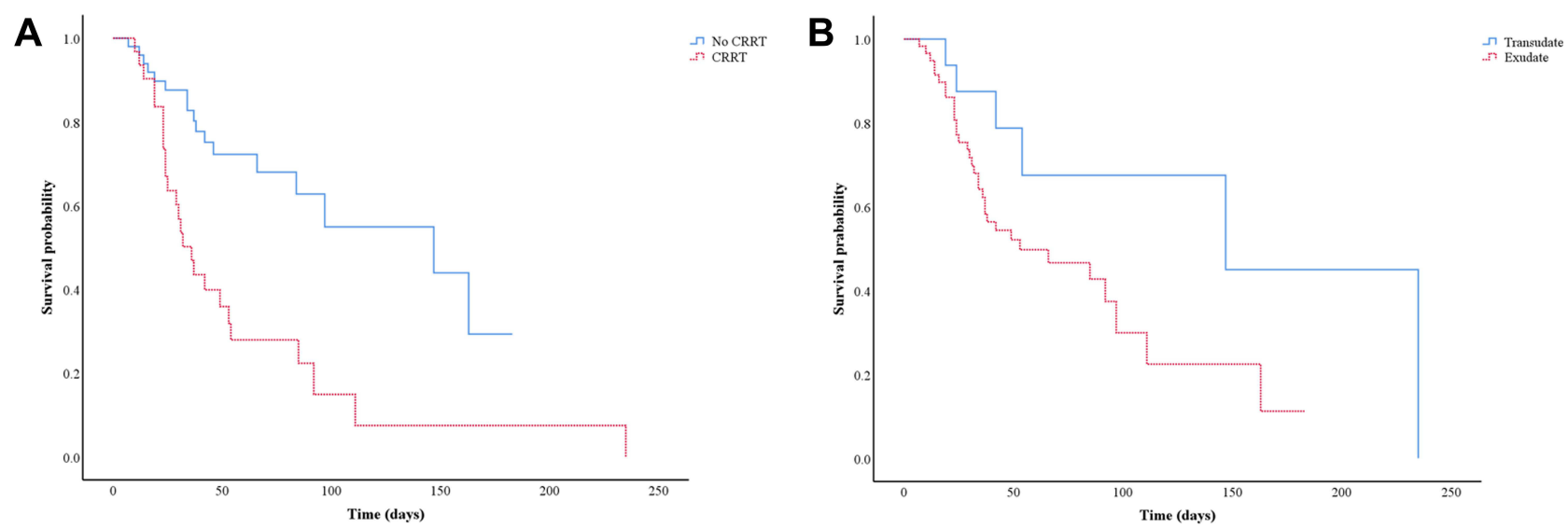

Figure I Kaplan-Meier survival analyses $(\mathbf{A})$ according to continuous renal replacement therapy $(C R R T, p<0.00$ I $)$ and $(\mathbf{B})$ the presence of transudate or exudate $(p=0.039)$.

pleural effusion have a higher 30-day mortality rate if they undergo catheter drainage instead of thoracentesis $(23.5 \%$ vs $18.6 \%) .{ }^{19}$ We also found that pleural drainage was associated with a high mortality rate among patients who required MV. Another study of 133 patients (MV was used for $82 \%$ of patients) revealed an in-hospital mortality rate of $49 \%,{ }^{20}$ which is similar to our result (51\%). In this setting, pleural drainage during MV is performed with the expectation of improvements in oxygenation or hemodynamic parameters, although its association with a high mortality rate suggests that pleural drainage during MV should be considered an indicator of serious comorbidity.
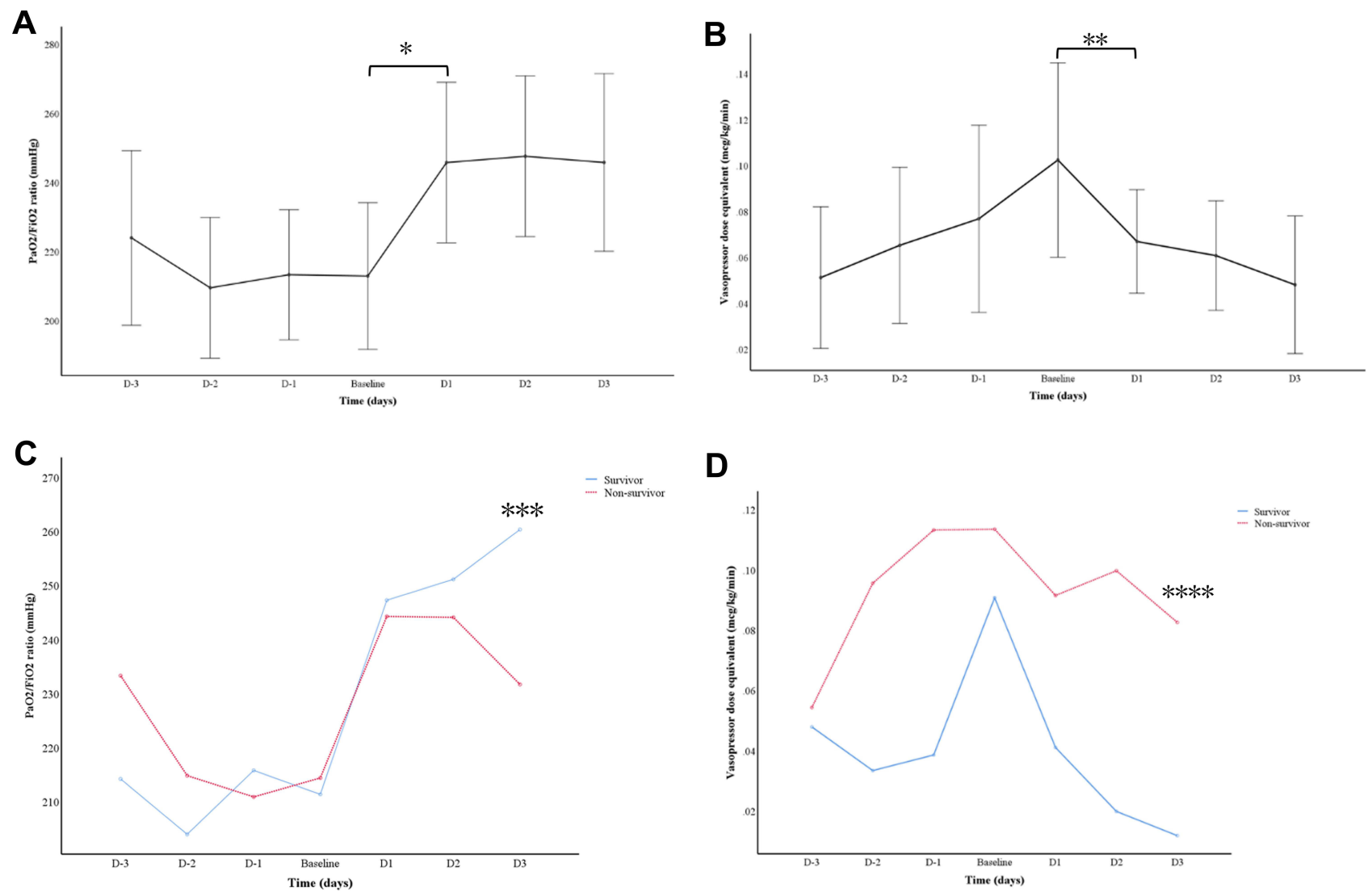

Figure 2 Changes in the $\mathrm{PaO}_{2} / \mathrm{FiO}_{2}$ ratio and vasopressor dose after pleural drainage. Changes are shown for $(\mathbf{A})$ the $\mathrm{PaO} / \mathrm{FiO} 2$ ratio $(\mathrm{p}=0.93 \mathrm{I})$, $(\mathbf{B})$ the vasopressor dose ( $p=0.0 \mathrm{II}$ ), (C) the $\mathrm{PaO}_{2} / \mathrm{FiO}_{2}$ ratio according to mortality, and (D) the vasopressor dose according to mortality. ${ }^{*}<0.00 \mathrm{I}$, the $\mathrm{PaO} / \mathrm{FiO}{ }_{2}$ ratio was significantly different between baseline and day I (DI). **p=0.03, the vasopressor dose was significantly different between baseline and $\mathrm{DI}$. $* * * \mathrm{p}=0.102$, the $\mathrm{PaO} / \mathrm{FiO} \mathrm{O}_{2}$ ratios on day 3 were 263 $( \pm 100)$ among survivors and $229( \pm 104)$ among non-survivors. $* * * * *=0.01 \mathrm{l}$, the vasopressor doses on day $3 \mathrm{were} 0.01 \mathrm{l}( \pm 0.04) \mu \mathrm{g} / \mathrm{kg} / \mathrm{min}$ among survivors and $0.08( \pm 0.18)$ $\mu \mathrm{g} / \mathrm{kg} / \mathrm{min}$ among non-survivors. 
Mortality after a diagnosis of pleural effusion is reportedly associated with the Acute Physiology And Chronic Health Evaluation II (APACHE II) score and malignancy. ${ }^{21}$ Previous studies of patients who were treated in the ICU, with/ without MV, have revealed mean APACHE II scores of $17-21,{ }^{20,22,23}$ which is approximately consistent with the median SOFA score of 11 in the present study. Thus, patients with severe illness who require CRRT and/or vasopressors at the time of pleural drainage may have a high risk of mortality. Similarly, pleural effusion is associated with an increased risk of mortality among patients who are undergoing maintenance hemodialysis, ${ }^{24}$ and fluid overload is an important contributor to the increased risk of mortality at RRT initiation. ${ }^{25}$ Therefore, CRRT use at the time of pleural drainage for patients who require MV indicates a poor prognosis. Patients with exudative pleural effusions also had a higher mortality rate (vs transudative effusions), which suggests that it is important to determine whether pleural effusions are exudative or transudative in this setting.

The $\mathrm{PaO}_{2} / \mathrm{FiO}_{2}$ ratio significantly improves at $24 \mathrm{~h}$ after starting pleural drainage, ${ }^{6,8,26}$ and our findings revealed no significant change in the $\mathrm{PaO}_{2} / \mathrm{FiO}_{2}$ ratio before starting pleural drainage. Thus, the significant improvement in the $\mathrm{PaO}_{2}$ $/ \mathrm{FiO}_{2}$ ratio is likely a result of pleural drainage. It is also interesting that the $\mathrm{PaO}_{2} / \mathrm{FiO}_{2}$ ratio remained relatively constant among survivors, but decreased by day 3 among non-survivors. The vasopressor dose also decreased significantly after $24 \mathrm{~h}$ of drainage, which might be related to improvements in hemodynamic parameters, including stroke volume. ${ }^{7}$ In critically ill patients, the maintenance of fluid balance is often achieved using diuretics or volume removal during CRRT. As pleural drainage can reduce the need for volume removal, which can lead to hypotension, dose of vasopressors can be reduced. Decline of dose of vasopressors was more remarkable among survivors. Therefore, it may be possible to predict outcomes among these patients based on changes in the $\mathrm{PaO}_{2} / \mathrm{FiO}_{2}$ ratio and vasopressor dose after starting pleural drainage. Therefore, maintenance of oxygenation and hemodynamic improvement by day 3 is more important than those by $24 \mathrm{~h}$ improvement in predicting mortality after pleural drainage in mv patients.

This study has several limitations. First, the retrospective nature of the study precluded collection of data regarding the reasons for performing pleural drainage. Second, although vasopressors and CRRT were important factors for mortality among mechanically ventilated patients who required pleural drainage, there is a possibility that nonsurvivors might have had greater severity, regardless of pleural drainage. Thrombocytopenia and higher C-reactive protein levels were common among the non-survivors and the length of hospital stay was significantly shorter in the nonsurvivors. Third, some results from the pleural fluid analysis were missing, which precludes a more comprehensive assessment of the removed pleural fluid. Therefore, well-designed prospective studies are needed to clarify whether the etiology of pleural effusions influences the risk of mortality among mechanically ventilated patients who require pleural drainage.

In conclusion, the present study revealed that among patients who required pleural drainage during MV, mortality was associated with the use of vasopressors, use of CRRT, and exudative pleural effusions. Furthermore, changes in the $\mathrm{PaO}_{2}$ $/ \mathrm{FiO}_{2}$ ratio and vasopressor dose by the third day of pleural drainage may be helpful for predicting the in-hospital mortality. Therefore, considering these factors may guide better prognostication and patient management.

\section{Data Sharing Statement}

The datasets used and analyzed during the current study are available from the corresponding author on reasonable request.

\section{Ethical Approval Statement}

This study was approved by the institutional review board of Chung-Ang University Hospital (2011-010-19341), and Ewha Womans University Mokdong Hospital (EUMC2021-02-004). The need for informed consent was waived due to the retrospective design.

\section{Funding}

There is no funding to report. 


\section{Disclosure}

The authors declare that they have no competing interests.

\section{References}

1. Bates D, Yang N, Bailey M, Bellomo R. Prevalence, characteristics, drainage and outcome of radiologically diagnosed pleural effusions in critically ill patients. Crit Care Resusc. 2020;22(1):45-52. doi:10.51893/2020.1.oa5

2. Bateman M, Alkhatib A, John T, Parikh M, Kheir F. Pleural effusion outcomes in intensive care: analysis of a large clinical database. $J$ Intensive Care Med. 2020;35(1):48-54. doi:10.1177/0885066619872449

3. Walden AP, Jones QC, Matsa R, Wise MP. Pleural effusions on the intensive care unit; hidden morbidity with therapeutic potential. Respirology (Carlton, Vic). 2013;18(2):246-254. doi:10.1111/j.1440-1843.2012.02279.x

4. Gilmartin JJ, Wright AJ, Gibson GJ. Effects of pneumothorax or pleural effusion on pulmonary function. Thorax. 1985;40(1):60-65. doi:10.1136/ thx.40.1.60

5. Mitrouska I, Klimathianaki M, Siafakas NM. Effects of pleural effusion on respiratory function. Can Respir J. 2004;11(7):499-503. doi:10.1155/ $2004 / 496901$

6. Goligher EC, Leis JA, Fowler RA, Pinto R, Adhikari NK, Ferguson ND. Utility and safety of draining pleural effusions in mechanically ventilated patients: a systematic review and meta-analysis. Crit Care. 2011;15(1):R46. doi:10.1186/cc10009

7. Wang Z, Cai QZ, Ban CJ, et al. Improved heart hemodynamics after draining large-volume pleural effusion: a prospective cohort study. BMC Pulm Med. 2018;18(1):62. doi:10.1186/s12890-018-0625-5

8. Razazi K, Thille AW, Carteaux G, et al. Effects of pleural effusion drainage on oxygenation, respiratory mechanics, and hemodynamics in mechanically ventilated patients. Ann Am Thorac Soc. 2014;11(7):1018-1024. doi:10.1513/AnnalsATS.201404-152OC

9. Razazi K, Boissier F, Neuville M, et al. Pleural effusion during weaning from mechanical ventilation: a prospective observational multicenter study. Ann Intensive Care. 2018;8(1):103. doi:10.1186/s13613-018-0446-y

10. Umbrello M, Mistraletti G, Galimberti A, Piva IR, Cozzi O, Formenti P. Drainage of pleural effusion improves diaphragmatic function in mechanically ventilated patients. Crit Care Resusc. 2017;19(1):64-70.

11. Colice GL, Curtis A, Deslauriers J, et al. Medical and surgical treatment of parapneumonic effusions: an evidence-based guideline. Chest. 2000;118 (4):1158-1171. doi:10.1378/chest.118.4.1158

12. Treml B, Rajsic S, Diwo F, Hell T, Hochhold C. Small drainage volumes of pleural effusions are associated with complications in critically Ill patients: a retrospective analysis. J Clin Med. 2021;10(11):2453. doi:10.3390/jcm10112453

13. Light RW, Macgregor MI, Luchsinger PC, Ball WC Jr. Pleural effusions: the diagnostic separation of transudates and exudates. Ann Intern Med. 1972;77(4):507-513. doi:10.7326/0003-4819-77-4-507

14. Light RW. Parapneumonic effusions and empyema. Proc Am Thorac Soc. 2006;3(1):75-80. doi:10.1513/pats.200510-113JH

15. Light RW, MacGregor MI, Ball WC Jr, Luchsinger PC. Diagnostic significance of pleural fluid pH and PCO2. Chest. 1973;64(5):591-596. doi:10.1378/chest.64.5.591

16. Goradia S, Sardaneh AA, Narayan SW, Penm J, Patanwala AE. Vasopressor dose equivalence: a scoping review and suggested formula. $J$ Crit Care. 2021;61:233-240. doi:10.1016/j.jcrc.2020.11.002

17. DeBiasi E, Puchalski J. Pleural effusions as markers of mortality and disease severity: a state-of-the-art review. Curr Opin Pulm Med. 2016;22 (4):386-391. doi:10.1097/MCP.0000000000000278

18. DeBiasi EM, Pisani MA, Murphy TE, et al. Mortality among patients with pleural effusion undergoing thoracentesis. Eur Respir J. 2015;46 (2):495-502. doi:10.1183/09031936.00217114

19. Hung TH, Tseng CW, Tsai CC, Hsieh YH, Tseng KC, Tsai CC. Mortality following catheter drainage versus thoracentesis in cirrhotic patients with pleural effusion. Dig Dis Sci. 2017;62(4):1080-1085. doi:10.1007/s10620-017-4463-8

20. Liang SJ, Tu CY, Chen HJ, et al. Application of ultrasound-guided pigtail catheter for drainage of pleural effusions in the ICU. Intensive Care Med. 2009;35(2):350-354. doi:10.1007/s00134-008-1314-2

21. Kookoolis AS, Puchalski JT, Murphy TE, Araujo KL, Pisani MA. Mortality of hospitalized patients with pleural effusions. $J$ Pulm Respir Med. 2014;4(3):184. doi:10.4172/2161-105X.1000184

22. De Waele JJ, Hoste E, Benoit D, et al. The effect of tube thoracostomy on oxygenation in ICU patients. J Intensive Care Med. 2003;18(2):100-104 doi: $10.1177 / 0885066602250358$

23. Talmor M, Hydo L, Gershenwald JG, Barie PS. Beneficial effects of chest tube drainage of pleural effusion in acute respiratory failure refractory to positive end-expiratory pressure ventilation. Surgery. 1998;123(2):137-143. doi:10.1016/S0039-6060(98)70250-8

24. Hamada S, Sano T, Nagatani Y, Tsukino M. Pleural effusion negatively impacts survival of patients undergoing maintenance hemodialysis. Pulmonology. 2019;25(1):58-60. doi:10.1016/j.pulmoe.2018.10.007

25. Bouchard J, Soroko SB, Chertow GM, et al. Fluid accumulation, survival and recovery of kidney function in critically ill patients with acute kidney injury. Kidney Int. 2009;76(4):422-427. doi:10.1038/ki.2009.159

26. Fysh ETH, Smallbone P, Mattock N, et al. Clinically significant pleural effusion in intensive care: a prospective multicenter cohort study. Crit Care Explor. 2020;2(1):e0070. doi:10.1097/CCE.0000000000000070 


\section{Publish your work in this journal}

The International Journal of General Medicine is an international, peer-reviewed open-access journal that focuses on general and internal medicine, pathogenesis, epidemiology, diagnosis, monitoring and treatment protocols. The journal is characterized by the rapid reporting of reviews, original research and clinical studies across all disease areas. The manuscript management system is completely online and includes a very quick and fair peer-review system, which is all easy to use. Visit http://www.dovepress.com/testimonials.php to read real quotes from published authors.

Submit your manuscript here: https://www.dovepress.com/international-journal-of-general-medicine-journal 